\title{
Advanced Thermal Simulator Testing: Thermal Analysis and Test Results
}

\author{
Shannon M. Bragg-Sitton, Ricky Dickens, David Dixon, Robert Reid, Mike \\ Adams, Joe Davis \\ ${ }^{I}$ NASA Marshall Space Flight Center, Nuclear Systems Branch / ER24, MSFC, AL 35812 \\ 256.544.6272, Shannon.M.Bragg-Sitton@nasa.gov
}

\begin{abstract}
Work at the NASA Marshall Space Flight Center seeks to develop high fidelity, electrically heated thermal simulators that represent fuel elements in a nuclear reactor design to support non-nuclear testing applicable to the development of a space nuclear power or propulsion system. Comparison between the fuel pins and thermal simulators is made at the outer fuel clad surface, which corresponds to the outer sheath surface in the thermal simulator. The thermal simulators that are currently being tested correspond to a SNAP derivative reactor design that could be applied for Lunar surface power. These simulators are designed to meet the geometric and power requirements of a proposed surface power reactor design, accommodate testing of various axial power profiles, and incorporate imbedded instrumentation. This paper reports the results of thermal simulator analysis and testing in a bare element configuration, which does not incorporate active heat removal, and testing in a water-cooled calorimeter designed to mimic the heat removal that would be experienced in a reactor core.
\end{abstract}

\section{INTRODUCTION}

At the NASA Marshall Space Flight Center (MSFC) Early Flight Fission Test Facility (EFF-TF) electric heaters are used to simulate the heat from nuclear fuel to test potential space fission power and propulsion systems. To allow early utilization, nuclear system designs must be relatively simple, easy to fabricate, and easy to test using nonnuclear heaters to closely mimic heat from fission. Specialized electric heaters are used to simulate the heat from nuclear fuel, allowing one to develop an understanding of individual components and integrated system operation without the cost, time and safety concerns associated with nuclear testing. Electric heaters have been used for this purpose in numerous terrestrial and space reactor test and development programs (e.g., Casal, 1980; Ott and McCulloch, 2004a; Suriano, 1993; Maslo and Hyer, 1976; Balashov et al., 2002). The thermal simulators (heaters) developed at the EFF-TF have been applied in a variety of space reactor concepts for power or propulsion applications, including heat pipe, direct gas, and liquid metal cooled reactor systems (Bragg-Sitton and Forsbacka, 2004; Godfroy, Kapernick and Bragg-Sitton, 2004; Vandyke et al., 2003; Vandyke, Martin and Houts, 2003). Thermal simulators previously designed and tested at the EFF-TF were constructed to meet the individual pin power levels and to roughly emulate the axial power distribution of specific reactor designs (Bragg-Sitton et al., 2007; Bragg-Sitton and Dickens, 2006). The current design effort focuses on developing thermal simulators that are fully instrumented and that mimic the transient nuclear fuel pin performance under typical transient operations.

Reactor designers at Los Alamos National Laboratory are currently working toward an optimized design for a fission surface power reactor system. During this design process, it is desirable for MSFC engineers to develop feasible design approaches and fabrication techniques that will enable rapid development of a high fidelity, instrumented simulator that will mimic the performance of the fuel pins in the optimized reactor design. To initiate this process a baseline liquid metal cooled reactor design is selected for current thermal simulator design and test activities. This "best estimate" design was derived from the only fission system that the United States has deployed for space operation, the Systems for Nuclear Auxiliary Power (SNAP) 10a reactor, which was launched in 1965 (Angelo and Buden, 1985). The resulting simulator design is discussed in Bragg-Sitton et al. (2007) and BraggSitton, Dickens and Dixon, (2007). In the absence of detailed requirements regarding the desired thermal simulator performance under static and transient conditions, a basic instrumented simulator design adhering to geometric and power requirements is developed. The primary objective of this exercise is to scope out potentially feasible design 
concepts and to work through any potential manufacturing issues. This simulator design does not seek to match static and dynamic fuel pin performance, but the design will be used as a baseline to initiate the detailed conceptual design for the next generation thermal simulators.

The simulator design currently under test adopts several key features not previously included in EFF-TF thermal simulators. This design incorporates imbedded instrumentation, can accommodate testing of various axial power profiles without full disassembly of the simulator (or removal from the reactor core simulator), and attempts to minimize simulator interference in the the coolant flow plenum. This simulator design concept is shown in Fig. 1 with key features identified. See Bragg-Sitton, Dickens and Dixon (2007) for further discussion of the simulator design, fabrication and assembly.

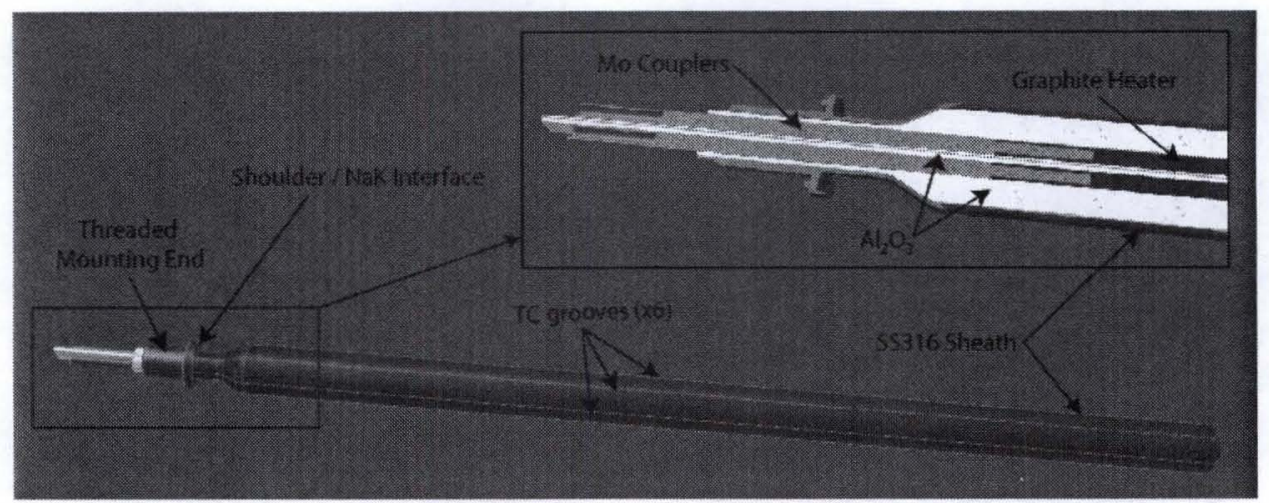

FIGURE 1. Instrumented thermal simulator design with reduced diameter feed through; TC grooves shown on the external sheath surface are mimicked on the $\mathrm{Al}_{2} \mathrm{O}_{3}$ insulator surface, allowing TCs to be installed between the insulator and sheath.

The current design allows up to 13 thermocouples (TCs, or fiber optics) to be installed in the simulator: 6 TCs on the sheath outer diameter (OD), $6 \mathrm{TCs}$ on the alumina $\left(\mathrm{Al}_{2} \mathrm{O}_{3}\right)$ insulator OD and one TC at the centerline of the heater element. TCs are employed on the simulator in all bare element and water-cooled calorimeter tests. Later testing in a single flow cell configuration, which emulates the liquid metal coolant flow path around a single simulator, will incorporate 3 fiber optics in the $\mathrm{Al}_{2} \mathrm{O}_{3}$ grooves to provide additional temperature distribution data. Fiber optic instrumentation can allow temperature measurement at intervals as small as one centimeter (Fielder, Klemer and Stinson-Bagby, 2004), but they have a delayed time response relative to TCs and, therefore, are not sufficient for real-time feedback control. Control algorithms for the NaK flow cell and future core array testing will continue to rely on TC instrumentation.

Testing of various axial power profiles that may be representative of different reactor conditions is enabled by designing the simulator to accommodate swap-out of the central heater element without full disassembly. In a liquid metal system, once the coolant has been introduced to the system it becomes very difficult to make any system modifications if they require disruption of the coolant barrier. Initial testing incorporates a constant diameter graphite heater element; a proposed design for an axially shaped graphite element is also presented in this paper.

In a fueled reactor design the fuel elements do not penetrate the inlet or outlet flow plenums; instead, they are anchored at a mounting plate at the plenum boundary. However, simulators require penetration through this region to allow power inlet/outlet to the individual pins. Power integration becomes more complex if the reactor design requires a pressure vessel or if the selected coolant is electrically conductive, as in liquid metal cooled systems. Integration is accomplished by extending the simulator sheath through the flow plenum, which can impact the flow dynamics (affecting the flow distribution and the overall system pressure drop). In attempt to minimize this impact the current design reduces the sheath OD as much as possible through the plenum while still allowing sufficient clearance for electrical connections and heater element removal. This design reduces the cross sectional area of the simulators in the plenum region to just one sixth the area that would be taken if the simulator OD is held constant through this region, reducing the impact on the flow distribution within the $\mathrm{NaK}$ plenum. Computational fluid dynamics (CFD) analysis will be employed in future analyses to quantify this benefit. 


\section{INITIAL BARE ELEMENT TESTING}

Bare element testing of the thermal simulator is conducted to ensure proper electrical performance of the simulator, to verify basic operation and to validate thermal analysis models before introducing additional variables. Significant care is taken in all tests to prevent any potential deformation of the simulator sheath due to unnecessary rapid heating, rapid cooling, or localized heating. In the actual core environment, the clad temperature is not expected to exceed $615^{\circ} \mathrm{C}(888 \mathrm{~K})$ with the fuel pin operating at $1075 \mathrm{~W}$, as predicted by SINDA/FLUINT (S/F) (Cullimore, Ring and Johnson, 2006). For test purposes the maximum allowed sheath temperature is set to $650{ }^{\circ} \mathrm{C}$, exceeding the maximum expected operating temperature in the full core array and staying well below deformation temperatures for stainless steel. The bare thermal simulator is tested up to $625 \mathrm{~W}$ in a $13 \mathrm{kPa}$ (100 torr) helium environment. The maximum tested power level is increased to the nominal operating levels of 860 and $1075 \mathrm{~W}$ (nominal and peak pin power in the baseline design) during later tests that incorporate active heat removal, but the same temperature limit is imposed. Steady state and transient testing are conducted for the bare simulator element to characterize basic simulator performance, to establish the maximum allowed heater power without exceeding the established temperature limitation for the simulator sheath and to determine the time response of the thermal simulator by measuring operating temperatures during transient conditions.

\section{Thermal Analysis}

Thermal analysis is performed using $\mathrm{S} / \mathrm{F}$ to determine anticipated simulator performance curves in a 100 torr helium environment. Major assumptions incorporated in the final simulator model developed in Thermal Desktop (Panczak et al. 2006) are summarized below:

- Power generation in copper leads based on total power and copper resistivity. Power delivered to pin is not reduced by amount delivered to wire (e.g. setting nominal pin power to $500 \mathrm{~W}$ means that $500 \mathrm{~W}$ is deposited in the graphite, and another $\sim 2-3 \mathrm{~W}$ will be deposited in the leads.

- No thermal expansion in solid elements.

- Calculation of radiation (Monte Carlo method) and conduction across gaps.

- Radiation from outer simulator sheath and from power leads to vessel (vacuum chamber) boundary.

- Power leads connected to boundary node with fixed temperature, allowing for conduction directly from graphite simulator.

- Simplified 3-piece implementation at reduced OD section; intermediate segment at average radius through taper.

- Contact conductance defined between all components physically touching; set to $10^{6} \mathrm{~W} / \mathrm{m}^{2}-\mathrm{K}$ for bonded materials and varied by using control parameters at the intersection of non-bonded materials.

- Variable (3-segment) emissivity on sheath surface.

- Temperature dependent thermal and optical properties where available.

- Fully parametric geometry description for all solid dimensions, including offsets for position.

Initial simulator check-out testing indicated significant temperature drops at either end of the simulator due to conduction along the power leads and the reduced OD power feed through section and into the alumina plug and stainless steel end cap at the non-powered end, in addition to radiative heat losses from both ends of the simulator. As a result the asymmetric ends of the simulator, power leads and all chamber walls are specifically described in the model to better predict the simulator temperatures. Several assumptions are made in the model with regard to material emissivities; environmental temperatures used in radiation calculations are based on experimental measurements (TCs located on the chamber wall)..

Initial $\mathrm{S} / \mathrm{F}$ analysis of the simulator assumed constant emissivity of the stainless steel sheath along the axis of the sheath, such that the temperature profile was relatively flat along the length of the simulator. However, significant oxidation was noted at the ends of the sheath after bare element testing was completed (Fig. 2), suggesting higher emissivity at the ends. Due to the noted oxidation the Thermal Desktop model was modified to define sheath emissivity in three axial sections. Literature suggests that the emissivity of stainless steel can vary from 0.16 to 0.85 depending on surface finish and oxidation. The original model applied a stainless steel emissivity of 0.55 at all temperatures and positions. In the modified model the central portion of the sheath applies linearly varying 
emissivity with temperature $(0.183$ at $0 \mathrm{~K}$ and 0.551 at $1000 \mathrm{~K})$. The emissivity is assumed to be elevated due to surface oxidation to 0.85 over the final $5 \mathrm{~cm}(2 ")$ at either end of the simulator.

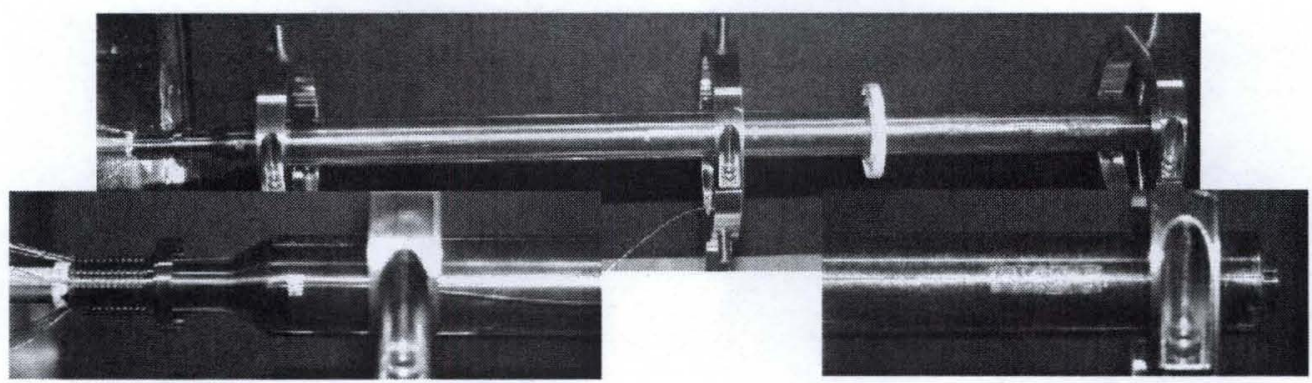

FIGURE 2. Thermal simulator sheath showing oxidation at the ends after bare element testing.

\section{Experimental Results}

The graphite heater element and graphite cement used to secure the power couplers were fully baked out / cured on during initial simulator testing. Subsequent testing indicates the expected axial temperature profile along the simulator, with the power input (bottle neck) end exhibiting a lower temperature due to the larger thermal mass in the longer power feed through structure relative to the end plug at the opposite end. The measured end-to-end temperature difference is approximately $20^{\circ} \mathrm{C}$ at $250 \mathrm{~W}$ and $40^{\circ} \mathrm{C}$ at 500 to $625 \mathrm{~W}$. Representative results are shown in the comparative analysis section. Plotted experimental results show error bars that correspond to the TC measurement error ( $\pm 2.5 \%$ of reading); however, the indicated error does not take into account error associated with TC positioning or other systematic errors that may be present.

The oxidation observed on the sheath (Fig. 2) is assumed to arise from differential temperature along the sheath surface. Oxidation is typically expected in regions of higher temperature, but observations indicate otherwise. These results suggest that impurities on the stainless steel or in the helium gas may result in oxidation of the sheath surface at a certain minimum temperature. All components are cleaned with ethyl alcohol prior to test, and the helium (He) used to fill the test chamber is designated as "ultra high purity (UHP)." Obtained from Sexton Supply, the UHP He has a guaranteed minimum purity of $99.999 \%$ and a maximum oxygen content of $3 \mathrm{ppm}$ (by volume); no additional purification of the gas is currently performed. The oxidation on the sheath surface may have burned off at the elevated temperatures along the central portion of the sheath but remained at the lower temperature ends of the sheath. Testing of the stainless steel stock material over carefully specified temperature intervals in the same test environment and employing the same cleaning procedures is required to further ascertain the source of the oxidation and the specific temperature range over which the oxidation occurs.

Initial transient testing of the thermal simulator considers two transient cases: $+25 \%$ (500 to $625 \mathrm{~W})$ and $-20 \%$ (625 $\mathrm{W}$ to $500 \mathrm{~W}$ ). Results indicate that the transient behavior is consistent across all test days, suggesting that the simulator performance is repeatable and that the components have been fully incurred during previous steady state testing; representative data are presented in the comparative analysis.

\section{Comparative Analysis}

A comparison of the experimental data and $\mathrm{S} / \mathrm{F}$ results suggests that the thermal analysis predicts the simulator sheath temperatures within a reasonable margin of error. Dashed lines in Fig. 3 correspond to the S/F results; data points shown with error bars correspond to experimental results from the bare simulator element.

Transient results provided in Fig. 4 correspond to TCs located at either end of the simulator sheath at $1.9 \mathrm{~cm}(0.75$ ", TC 7) and $41.1 \mathrm{~cm}\left(16.25 "\right.$, TC 12) and near the mid-section of the sheath at $16.5 \mathrm{~cm}\left(6.5^{\prime \prime}\right.$, TC 9) and $26.7 \mathrm{~cm}$ (10.5", TC 10). All positions are measured from the start of the heated zone at the shoulder of the sheath assembly (power input end). Transient S/F results are shown for the central portion of the sheath (data at 16.9 and $27.9 \mathrm{~cm}$, labeled as "mid-sheath," predict approximately the same temperature) and near the ends of the sheath at $1.9 \mathrm{~cm}$ and $41.1 \mathrm{~cm}$. The transient is initiated from steady state operation at $500 \mathrm{~W}(+25 \%$ power) at time zero in Fig. 4(a). 
After achieving a new steady state at the elevated power level of $625 \mathrm{~W}$ the second transient $(-20 \%$ power) is initiated at what is shown as time zero in Fig. 4(b). The analysis and experimental data indicate that the simulator temperature returns to the original temperature after a transient, demonstrating that the simulator is not permanently modified by operation at increased power and temperature.

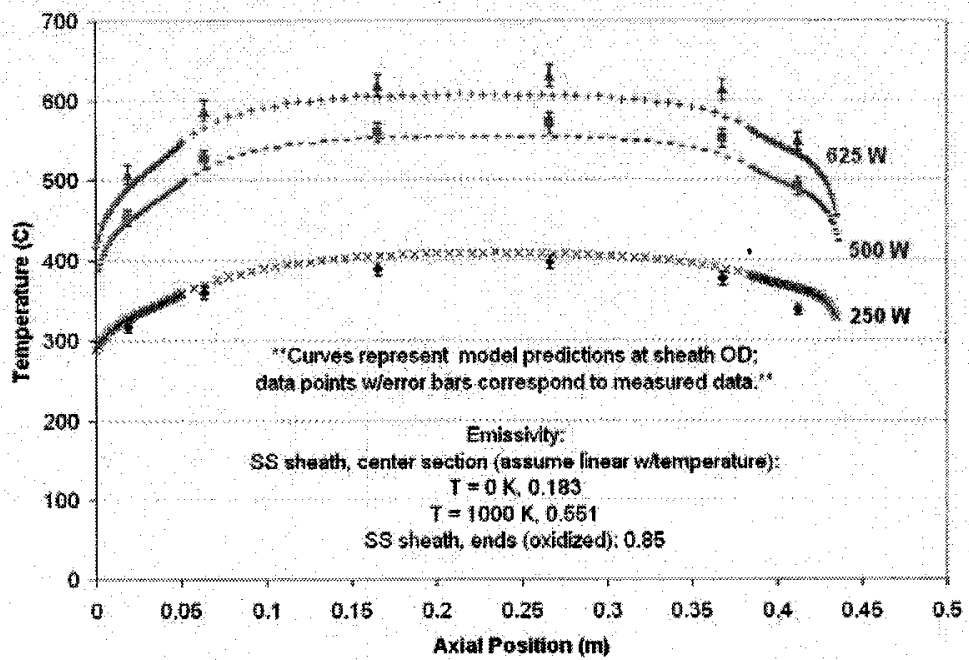

FIGURE 3. Analysis and experimental results for steady state testing of the instrumented simulator, with inclusion of axially varying surface emissivity.

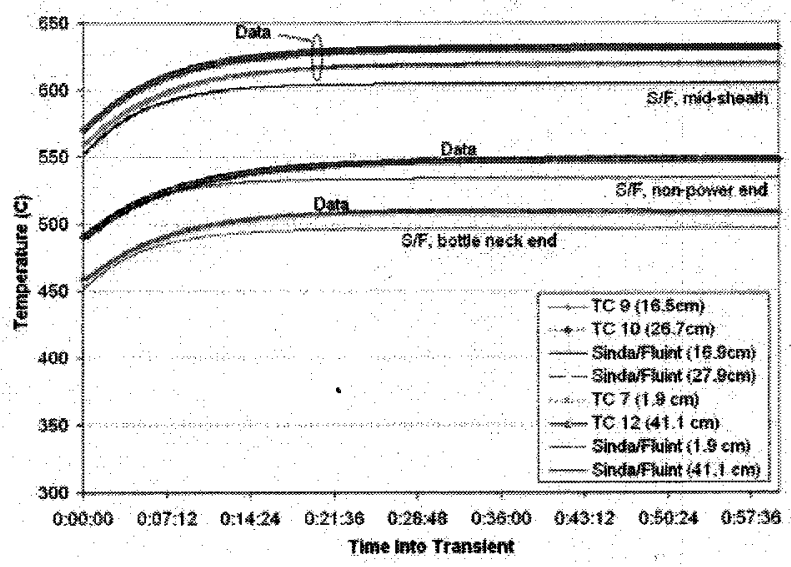

(a)

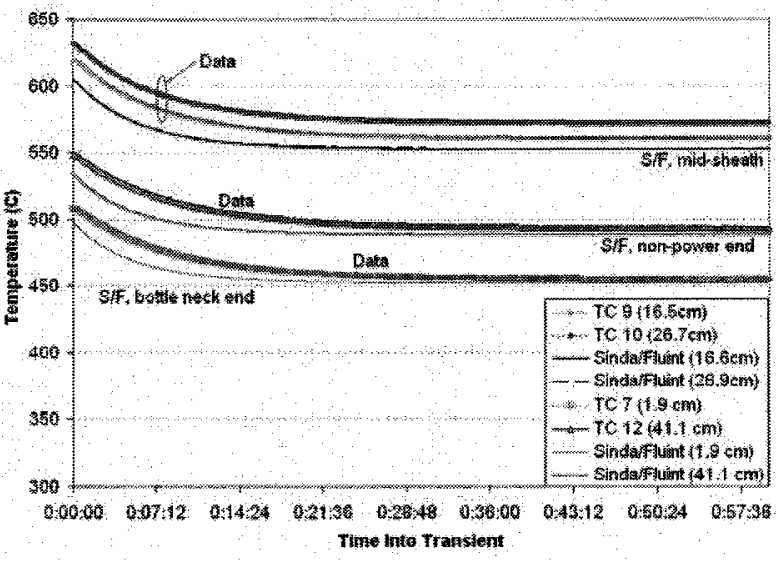

(b)

FIGURE 4. Transient thermal analysis and test data for (a) $25 \%$ power increase from 500 to $625 \mathrm{~W}$ and (b) $20 \%$ power decrease from 625 to $500 \mathrm{~W}$.

Experimental results suggest a simulator response time of approximately $25 \mathrm{~min}$ for the applied transients with no active heat removal from the simulator. Thermal analysis predicts slightly more rapid heating/cooling of the element than observed experimentally (approximate $20 \mathrm{~min}$ time constant), suggesting that some of the parameters and material properties used in the model may differ somewhat from the actual configuration. Properties such as thermal conductivity, density and specific heat are taken from the literature, and material emissivities are estimated from literature and the visible condition of the emitting material.

In the experimental data, TCs 9 and 10 are located symmetrically about the axial midpoint of the simulator. However, TC 10 is consistently hotter in the transient data; this is also true of the steady state data, in which the power lead end of the simulator is approximately $40^{\circ} \mathrm{C}$ cooler at the power inlet end at 500 and $625 \mathrm{~W}$. The slight 
asymmetry at the central portion of the sheath is likely due to the larger heat sink at the power feed end of the simulator, causing the temperature to be slightly more depressed at that end. Additionally, the power feed end is "open," allowing radiation heat loss from the element in addition to conductive losses (along the sheath and through the power leads), although the view factor from the heater element to the cool chamber wall is relatively small due to the extended bottle shape. Differences in the measured temperatures for TCs 9 and 10 may also be due to TC placement differences (e.g. TC 10 may have better contact with the simulator sheath than TC 9, causing TC 10 to register a more accurate sheath temperature). Error bars on the measured temeprature are not included in transient data to simplify the presentation of the results. The predicted temperature along the central portion of the sheath remains relatively flat despite modifications to the thermal analysis to include end effects and variable axial emissivity along the sheath.

\section{CALORIMETER TESTING}

Element testing inside a water cooled calorimeter allows characterization of the simulator thermal response with active heat removal. The tested calorimeter consists of an annular shell and cover. Heat is transferred by radiation, gas conduction, and natural convection from the sheath surface to a stream of water that flows through the annular gap between the shell and the cover. The shell is separated from the sheath by a gas-fill of fixed thickness; initial testing employs high purity helium (He) in all tests. The calorimeter is constructed from four identical $10.2 \mathrm{~cm}$ (4.0") copper sections that allow water flow through an annular gap to extract heat from the thermal simulator. Thermal isolation of each section of the calorimeter is provided by thin alumina rings. The amount of heat removed by each leg of the calorimeter can be determined by measuring the mass flow rate of water through that section and the inlet and outlet water flow temperatures; the associated calculation is given in Eq. (1):

$$
Q=\dot{m} C_{p}\left(T_{\text {out }}-T_{i n}\right)
$$

The water flow to the calorimeter is provided by a single inlet flow line that is split into four branches at a manifold located outside the test chamber, just upstream of the flow measurement instrumentation. The outlet flow from each calorimeter section goes to a flow manifold inside the chamber, allowing a single outlet line to exit through the chamber feed through. Each section is independently instrumented with a flow meter and inlet and outlet TCs to allow calculation of the power removed by that section. All imbedded simulator instrumentation is maintained in the calorimeter test configuration.

The initial calorimeter test configuration employs four $1288 \mathrm{ml} / \mathrm{min}$ rotameters with stainless steel floats; the associated uncertainty in the flow meter measurement is $\pm 2 \%$ full scale (FS). The inlet and outlet water temperatures are measured at T-junctions that are $4.8 \mathrm{~cm}\left(1.9^{\prime \prime}\right)$ from the calorimeter body on each section of the calorimeter. Temperature measurements are acquired using type $\mathrm{K}$, special limits of error (SLE) TCs that have a reported $\pm 1.1^{\circ} \mathrm{C}$ uncertainty (or $0.4 \%$, whichever is greater). However, detailed uncertainty analysis for this configuration indicates an uncertainty in the enthalpy removed by the calorimeter of $17.7 \%( \pm 200 \mathrm{~W})$ for an input electric power of $1075 \mathrm{~W}$ to as much as $58.2 \%( \pm 58 \mathrm{~W})$ for an electric power of $100 \mathrm{~W}$. Uncertainty analysis of four potential instrumentation configurations led to the selection of alternate flow rate and temperature measurement instrumentation for the calorimeter based on minimizing both cost and uncertainty. This modified configuration replaces the water TCs with International Electrotechnical Commission (IEC) 541 resistance temperature detectors (RTDs) $\left( \pm 0.2^{\circ} \mathrm{C}\right.$ uncertainty) and modifies the rotameters to utilize glass floats such that they are capable of measuring $514 \mathrm{ml} / \mathrm{min}( \pm 2 \% \mathrm{FS})$. Uncertainty analysis for this configuration indicates uncertainties of 3 to $5 \%$ in the enthalpy removed by the calorimeter for input electrical power of $1075 \mathrm{~W}$ down to $500 \mathrm{~W}$. Results presented below correspond to the enhanced configuration.

To improve the radiative coupling of the thermal simulator to the copper calorimeter, the inner surface of the calorimeter was treated with a chemical blackening agent produced by Caswell Plating (A324) to increase the material emissivity. A324 "swab-on copper blackener" is a corrosive liquid composed of phosphoric acid and selenious acid that acts as a chemical conversion finish for copper and its alloys. The approximate emissivity of shiny copper is 0.15 ; the blackened copper has an emissivity of approximately 0.9 .

A photo of the assembled calorimeter (initial configuration) is shown inside the test chamber in Fig. 5. The four flow inlet lines enter at the bottom of each calorimeter segment (such that water is introduced to the initial calorimeter 
section at the start of the heated zone) and flow outlets are at the top of the calorimeter. TCs located at the Tjunction at both the flow inlet and outlet are shown in the assembly photo. The outlet flow manifold can be seen downstream of the outlet TCs on the right side of the photo.

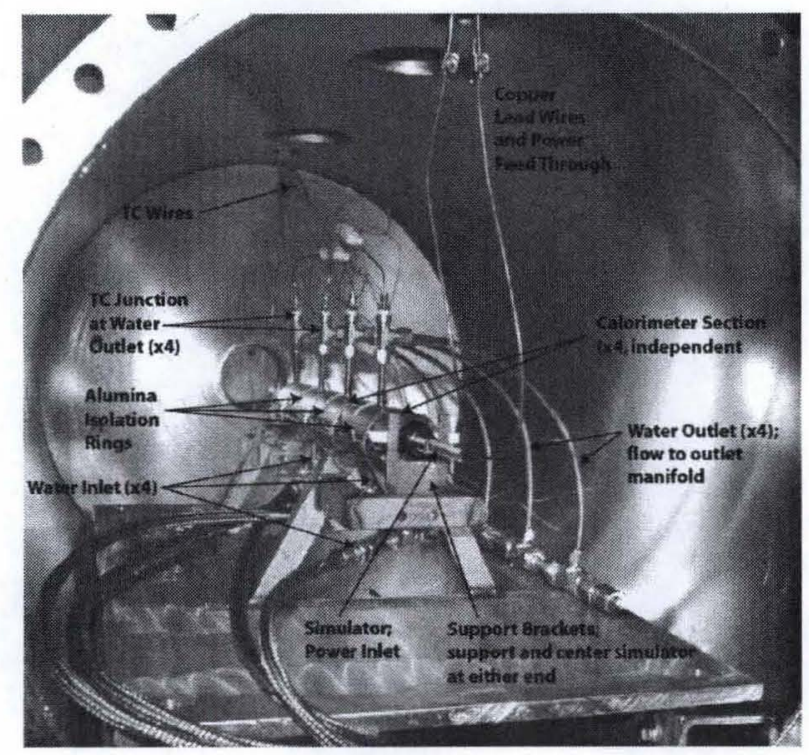

FIGURE 5. Assembled calorimeter hardware.

\section{Thermal Modeling}

The bare element simulator model was used as a basis for the calorimeter model with a few minor changes to the configuration taken into account. Prior to installation in the calorimeter the simulator sheath was cleaned to remove oxidation at the ends. Until the simulator is completely removed from the calorimeter for further visual analysis the thermal analysis model assumes that the sheath emissivity is consistent along the heated length regardless of axial position due to the more even heat removal along simulator axis with the calorimeter in place (although the subdivision into three sections is maintained for potential future use). Increased emissivity due to oxidation on the end sections of the sheath that are not covered by the calorimeter (bottle neck and end cap regions) is incorporated into the analysis. Additional assumptions made in the simulator / calorimeter model are summarized below:

- Radiation from outer calorimeter wall and from power leads to vessel boundary. Radiation included from sheath/end components to both vessel wall and calorimeter inner surface.

- Fully parametric geometry description for all solid dimensions, including offsets for position in calorimeter. Flow areas, hydraulic diameters, etc. are all calculated based on parameters specified in the Thermal Desktop symbol manager.

- Independent water flow submodels, allowing different inlet temperature/flow rate conditions for each calorimeter subsection.

The simulator is housed within the sectioned copper calorimeter, separated by the thin alumina rings discussed previously. As in the bare element model, the calorimeter thermal model incorporates several assumptions that are made based on literature, physical appearance of materials, assembly tolerances etc. Many of these assumptions cannot be verified experimentally given the small amount of measured temperature data available. Key assumptions include contact conductance between the alumina isolating rings and the simulator sheath, contact conductance between the graphite heater element and the alumina end plug, sheath emissivity and emissivity of the inner, blackened surface of the copper calorimeter. Based on previous model validation for the bare element the sheath emissivity is set to that for the central portion of the sheath $(0.183$ at $0 \mathrm{~K}$ and 0.551 at $1000 \mathrm{~K})$. Literature indicates that the emissivity of shiny copper is approximately 0.15 ; this value is adopted for all copper surfaces with the exception of the blackened copper, which is assumed to have an emissivity of 0.9 . Contact conductance parameters are assessed over a wide range in the comparative analysis section. 
In the final thermal model configuration, the contact conductance between the leads and the fuel pin is set to $10^{4}$ $\mathrm{W} / \mathrm{m}^{2}-\mathrm{K}$ and the boundary temperature at the end of the power leads is set to $343 \mathrm{~K}$ (50 degrees above room temperature). On the opposite end of the simulator the heated section is terminated with an alumina end plug and a stainless steel end cap. The alumina end plug is in contact with but is not bonded to the graphite heater element. Iterative calculations at $500 \mathrm{~W}$ heater power given a contact conductance for the end plug of 0.1 to $10^{6} \mathrm{~W} / \mathrm{m}^{2}-\mathrm{K}$ suggests that an appropriate value may be $10^{3} \mathrm{~W} / \mathrm{m}^{2}-\mathrm{K}$ or less. Any higher value causes an upturn in the temperature near the end of the simulator, which is not physical. However, any value from 0.1 to $10^{3} \mathrm{~W} / \mathrm{m}^{2}-\mathrm{K}$ provides a reasonable estimate of the end temperatures relative to that measured; additional temperature measurements are required to further assess the correct conductance value. Comparative analysis below adopts a contact conductance of $0.1 \mathrm{~W} / \mathrm{m}^{2}-\mathrm{K}$.

Finally, each of the four copper calorimeter sections is separated by a thin alumina ring such that each section is isolated electrically and thermally. These rings make a slip fit over the simulator sheath and fit around the ends of the adjacent sections of the calorimeter. Iterative calculations vary the contact conductance between the sheath and the $\mathrm{Al} 2 \mathrm{O} 3$ rings from $1 \mathrm{~W} / \mathrm{m}^{2}-\mathrm{K}$ to $400 \mathrm{~W} / \mathrm{m}^{2}-\mathrm{K}$ for an input power of $500 \mathrm{~W}$ to the thermal simulator. Results for $10,100,200$ and $400 \mathrm{~W} / \mathrm{m}^{2}-\mathrm{K}$ and a blackened copper emissivity of 0.8 and 0.9 (both at $100 \mathrm{~W} / \mathrm{m}^{2}-\mathrm{K}$ ) are presented below. Results suggest that a value of $100 \mathrm{~W} / \mathrm{m}^{2}-\mathrm{K}$ for the alumina to sheath contact conductance may be appropriate, but it is difficult to ascertain this with any certainty due to error in the measured temperatures (error bars not shown in plot) and TC placement. Because measured temperature data is limited and no TCs fall within the region of the alumina spacers it is difficult to determine if the dips in temperature shown in the thermal analysis are physical. The magnitude of the dips increases with increased contact conductance, with the axial profile relatively continuous given a contact conductance of $10 \mathrm{~W} / \mathrm{m}^{2}-\mathrm{K}$ or less.

\section{Experimental Results and Comparative Analysis}

The measured simulator sheath temperatures for the upgraded calorimeter configuration are plotted with the corresponding thermal analysis in Fig. 6. Transient response testing (not shown) indicates that the calorimeter responds in a first order fashion with a $\sim 1000$-s time constant. One may note that only four sheath temperatures are shown for the measured data; in addition to TC 5 (located at $36.8 \mathrm{~cm}\left(14.5^{\prime \prime}\right)$ ) that failed in previous testing TC 2 (located at $6.35 \mathrm{~cm}\left(2.5^{\prime \prime}\right)$ ) also failed to operate after modifications were implemented on the calorimeter hardware. As noted above, a detailed iterative analysis was conducted at $500 \mathrm{~W}$ simulator operation to determine the most appropriate value for the contact conductance between the alumina rings and simulator sheath (Fig. 6(a)). In this comparison both 100 and $200 \mathrm{~W} / \mathrm{m}^{2}-\mathrm{K}$ provided reasonable representation of the measured simulator performance; however, the reduced dips in the predicted temperatures observed for a contact conductance of $100 \mathrm{~W} / \mathrm{m}^{2}-\mathrm{K}$ are believed to be more physically representative of the actual sheath temperatures. Comparison of modified emissivity for the blackened copper $(0.8$ or 0.9$)$ is shown for this contact conductance, although analysis results indicate little difference for these emissivity values. The $100 \mathrm{~W} / \mathrm{m}^{2}-\mathrm{K}$ contact conductance and blackened copper emissivity of 0.9 are adopted for the remainder of the thermal analysis presented in Fig. 6(b).

Fig. 7 shows a comparison of the electric power delivered to the heater element and the calculated power removed by the calorimeter. The corresponding heat balance, which calculates the percent difference between the power delivered and the power removed, indicates that the input power exceeds that removed by approximately $5 \%$ at the higher power levels tested. The corresponding uncertainty analysis indicates error in the calculated power removed by the calorimeter of approximately 3 to $5 \%$ over the power levels of interest (500 to $1075 \mathrm{~W}$ ). Spikes in the calculated power and heat balance correspond to adjustments in the water mass flow rate.

\section{DISCUSSION AND FUTURE WORK}

The instrumented thermal simulator development and testing conducted to date has demonstrated the fabrication capability and associated complications for the various simulator components and basic simulator operation with and without active heat removal. Additional testing proposed for the current simulator design may include testing with active heat removal in a geometrically accurate, flowing liquid metal environment and testing with a shaped graphite heater element to demonstrate performance with an axially varying power profile. 


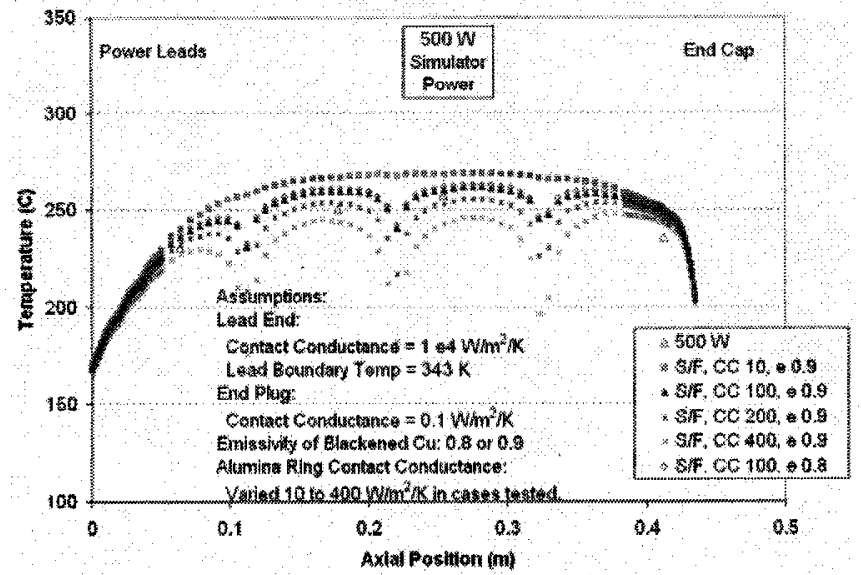

(a)

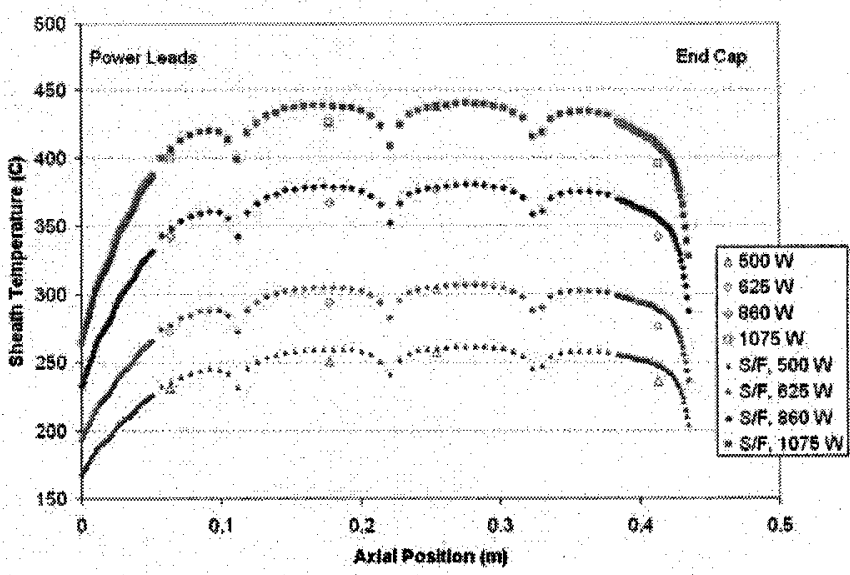

(b)

FIGURE 6. Comparison of thermal analysis and experimental data acquired for the modified calorimeter configuration: (a) Analysis of the effect of contact conductance between the alumina spacer rings and the simulator sheath at $500 \mathrm{~W}$, and (b) comparison at selected power levels using optimal parameters from iterative analysis.

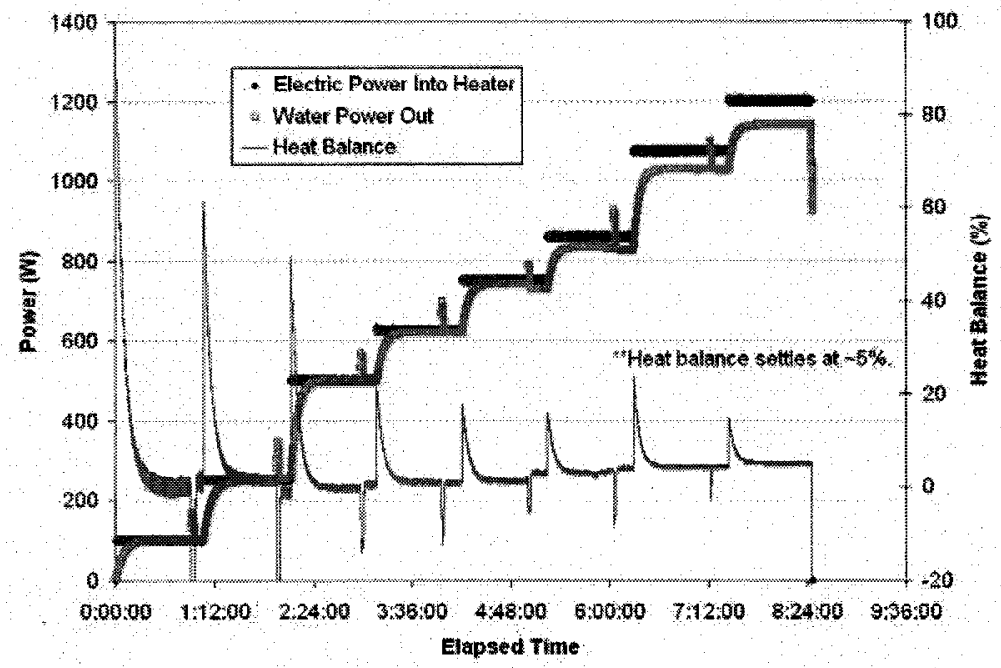

FIGURE 7. Inlet electric power and calculated calorimeter power and associated heat balance.

\section{Single Flow Cell Test Article}

Additional thermal simulator testing will be conducted in a single flow cell test article (SFCTA) that represents the liquid metal $(\mathrm{NaK})$ flow path around a single thermal simulator in the baseline conceptual reactor design. Heat deposition from neighboring fuel (simulator) elements will be simulated using cartridge heaters to achieve the appropriate boundary conditions. The objective of this test is to study thermal simulator performance with active heat removal in a geometrically accurate flowing $\mathrm{NaK}$ environment. Simulator response to transients on the primary or secondary side of the system (e.g. power changes to the heater or variation in coolant flow rate or inlet temperature) are of key interest in the test matrix being developed. Simulator response will be studied by monitoring temperatures along the thermal simulator sheath using imbedded TCs, fiber optics installed between the simulator sheath and the internal alumina insulator components, and TCs installed in the SFCTA structure. In addition to temperature the pressure drop along the flow channel is of particular interest to reactor designers. The core pressure drop is significant to the overall system design and the test article may be used to validate model calculations. The measured pressure drop will only incorporate the axial flow channel and will not make any attempt to assess pressure drop in the inlet or outlet flow plenum, as neither is prototypic to the full core design. 


\section{Shaped Heater Designs}

An alternate, shaped graphite element design has been developed to achieve the power profile predicted for the baseline reactor design employed in the current simulator development. Initial constraints set the maximum heater OD at $0.874 \mathrm{~cm}(0.344 ")$, consistent with the current constant diameter heater element. Previous shaped graphite element designs produced by and tested at the EFF-TF utilized a simplified element shape in which a square cut-out was removed from the OD along the central $10.2 \mathrm{~cm}\left(4^{\prime \prime}\right)$ of the element. Although this design results in a rough cosine axial power distribution it was not designed to mimic any particular reactor power profile. Shaping of the element from the inner diameter (ID) is preferable to removing material from the OD, as it maintains constant thermal coupling to the next layer of the simulator buildup (e.g. alumina insulator sleeve) at all axial and azimuthal positions. Calculation of the desired element ID is based on an approximate graphite resistivity of $30 \mu \Omega-\mathrm{m}$, although the graphite composition and corresponding resistivity may vary based on the specific material selected for the heater element. Fig. 8 shows a conceptual shaped element design. A circular shape is selected for the open area cross section to maintain a constant graphite thickness at each axial position, reducing the potential of localized heating at any azimuthal position and achieving a maximum graphite thickness around the circumference.

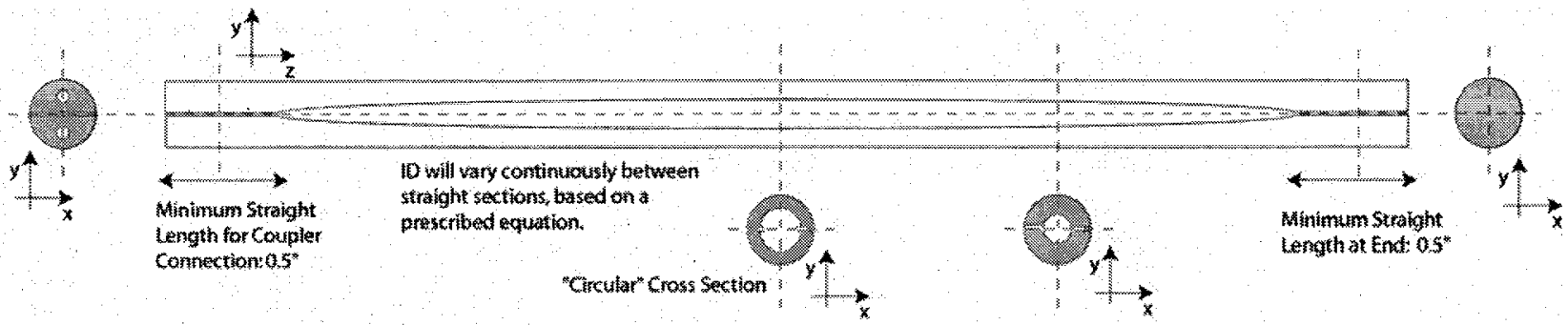

FIGURE 8. Conceptual shaped graphite element design.

In calculating the desired ID at each axial location the element is discretized into $1.27 \mathrm{~cm}\left(0.5^{\prime \prime}\right)$ lengths to determine an approximate shape; a solver routine in Microsoft Excel is employed to iteratively solve for the element ID to obtain the desired cosine power shape. The smoothed profile determined in Excel is shown in Fig. 9. A polynomial fit to the resulting ID is determined using a polynomial fit routine in MatLab that computes a solution by using a least squares fitting routine. The resulting equation is provided to the engineering designer to develop a design that can be used by a vendor for fabrication.

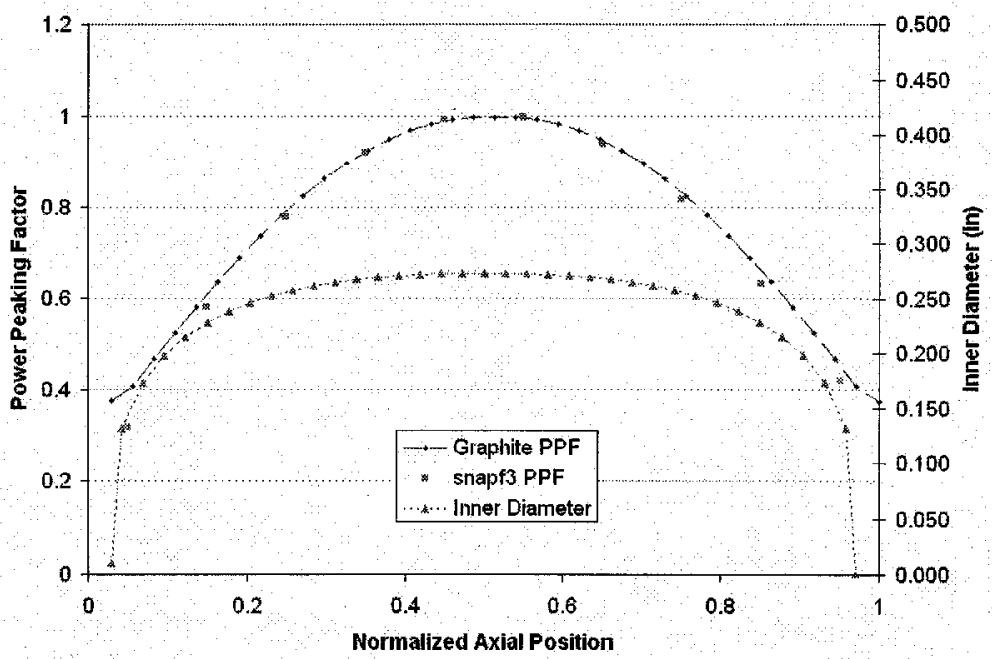

FIGURE 9. Proposed shaped graphite element ID design with approximate power peaking factor for the graphite and the baseline reactor design. 
Several options are being considered for the formation of the proposed graphite element design. Due to the complexity of the interior shape of the graphite it cannot be cast in a single element and subsequently be machined to form the desired shaping. Instead, the element could be cast over a mandrel (non-conductive or one that can be removed by chemical processes) or fabricated in separate components. Options are currently being discussed with potential vendors.

\section{CONCLUSIONS}

Lessons learned from the simulator development presented here are currently being taken into account in the development of a high fidelity simulator design. The next generation simulator will be based on the current liquid metal cooled reactor core design provided by engineers at LANL and will be designed such that its static and dynamic performance will better mimic that of a fueled element relative to previous simulator designs.

\section{NOMENCLATURE}

$\mathrm{Q}=$ heat removed $(\mathrm{W})$

$\mathrm{m}=$ water mass flow rate $(\mathrm{kg} / \mathrm{s})$

$\mathrm{C}_{\mathrm{p}}=$ specific heat capacity of water $(\mathrm{J} / \mathrm{kg}-\mathrm{K})$

$\mathrm{T}_{\text {out }}=$ outlet water temperature $(\mathrm{K})$

$\mathrm{T}_{\text {out }}=$ inlet water temperature $(\mathrm{K})$

\section{ACKNOWLEDGMENTS}

The work described within this report was supported by NASA, in whole or part, as part of the agency's technology development and evaluation activities. Any opinions expressed are those of the author(s) and do not necessarily reflect the views of NASA.

\section{REFERENCES}

Balashov, S.M., Videneev, E.N., Veresov, A.V., Zorichev, V.V., Mal'tsev, B.K., and Smolin, V.N., "Electrical Heaters for Experimental Installations and Nuclear Power Stations," Thermal Engineering, 49(5), pp. $377-381$ (2002).

Bragg-Sitton, S.M. and Dickens, R., "Thermal Simulator Development: Non-Nuclear Testing of Space Fission Systems," in Transactions of the American Nuclear Society, American Nuclear Society, Vol. 95, La Grange Park, IL, 2006, pp. 907-908.

Bragg-Sitton, S.M., Dickens, R, Dixon, D.D., "High Fidelity Thermal Simulators for Non-Nuclear Testing: Analysis and Initial Results," in proceedings of the Space Nuclear Conference (SNC'07), American Nuclear Society, La Grange Park, IL, 2007, pp. 336-345.

Bragg-Sitton, S.M., Dickens, R, Dixon, D.D., Kapernick, R., Adams, M. and Davis, J., "Development of High Fidelity, Fuel-Like Thermal Simulators for Non-Nuclear Testing," in Proceedings of Space Technology and Applications International Forum (STAIF-2007), edited by M.S. El-Genk, AIP Conference Proceedings 880, Melville, New York, 2007, pp. 605-614.

Bragg-Sitton, S.M. and Forsbacka, M., "Application of a Virtual Reactivity Feedback Control Loop in Non-Nuclear Testing of a Fast Spectrum Reactor," in proceedings of International Congress on Advanced Nuclear Power Plants (ICAPP-2004), American Nuclear Society, La Grange Park, IL, 2004, pp. 2259-2268.

Casal, V., "Design of High-Performance Fuel Pin Simulators for Thermodynamic Experiments with Nuclear Fuel Elements," Nuclear Technology, 47(1), 153-162 (1980).

Cullimore, B.A., Ring, S.G. and Johnson, D.A., SINDA/FLUINT 5.0, Cullimore \& Ring Technologies, Inc., Littleton, CO, 2006.

Fielder, R.S., Klemer, D. and Stinson-Bagby, K., "High-Temperature Fiber Optic Sensors, an Enabling Technology for Nuclear Reactor Applications," in proceedings of the 2004 International Congress on Advances in Nuclear Power Plants (ICAPP2004), Pittsburgh, PA, 2004, pp. 2295-2305.

Godfroy, T.J., Kapernick, R., and Bragg-Sitton, S.M., "Thermally Simulated 32kW Direct-Drive Gas-Cooled Reactor: Design, Assembly and Test," in proceedings of Space Technology and Applications International Forum (STAIF-2004), edited by M.S. El-Genk, AIP Conference Proceedings 699, Melville, New York, 2004, pp. 757-763.

Maslo, R.M., "Hybrid Simulation of a Nuclear Fuel \& Electric Heater Pin," in proceedings of the $8^{\text {th }}$ AICA Congress, edited by L. Dekker, North-Holland Publishing Company, Amsterdam, Netherlands, 1976, pp. 795-803.

Ott, L.J. and McCulloch, R, "Overview of Fuel Rod Simulator Usage at ORNL;" Space Technologies and Applications International Forum (STAIF-2004), edited by M.S. El-Genk, AIP Conf Proc 699, Melville, New York, 2004, pp. 703-712. 
Panczak, T.D., Ring, S.G., Welch, M.J. and Johnson, D.A., Thermal Desktop 5.0, Cullimore \& Ring Technologies, Inc., Littleton, $\mathrm{CO}, 2006$.

Suriano, M., "TOPAZ-II Program: Initial US Demonstration Test Results on the TOPAZ-II Thermionic Space Nuclear Power System," in Proceedings of the $28^{\text {th }}$ Intersociety Energy Conversion Engineering Conference, American Chemical Society, Washington, D.C., 1993, pp. 855-861.

VanDyke, M. K., Houts, M.G., Godfroy, T.J., Dickens, R., Martin, J.J., Poston, D.I., Salvail, P., and Carter, R. (2003), "Test Facilities in Support of High Power Electric Propulsion Systems," in proceedings of Space Technology and Applications International Forum (STAIF-2003), edited by M.S. El-Genk, AIP Conference Proceedings 654, Melville, New York, 2003, pp. 451-456.

VanDyke, M.K., Martin, J.J., and Houts, M.G., Overview of Nonnuclear Testing of the Safe, Affordable 30-kW Fission Engine Including End-to-End Demonstrator Testing, NASA/TM-2003-212930, NASA Marshall Space Flight Center, Huntsville, $\mathrm{AL}, 2003$. 